\title{
BLOW-UP ON THE BOUNDARY: A SURVEY
}

\author{
MAREK FILA \\ Department of Mathematical Analysis, Comenius University \\ Mlynská dolina, 84215 Bratislava, Slovakia \\ JÁN FILO \\ Institute of Applied Mathematics, Comenius University \\ Mlynská dolina, 84215 Bratislava, Slovakia
}

Introduction. In this survey we review some results on blow-up of solutions of the problem

$$
\begin{array}{lr}
\frac{\partial u^{m}}{\partial t}=\Delta u, & x \in \Omega, t>0, \\
\frac{\partial u}{\partial \nu}=u^{p}, & x \in \partial \Omega, t \geq 0, \\
u(x, 0)=u_{0}(x)>0, & x \in \bar{\Omega}, \\
\frac{\partial u_{0}}{\partial \nu}=u_{0}^{p}, & x \in \partial \Omega,
\end{array}
$$

where $m, p>0$ and $\Omega$ is either a smoothly bounded domain in $\mathbb{R}^{N}$ or $\Omega=\mathbb{R}_{+}^{N}=$ $\left\{\left(x_{1}, x^{\prime}\right): x^{\prime} \in \mathbb{R}^{N-1}, x_{1}>0\right\}, \nu$ is the outward normal.

Over the past two decades this problem has received considerable interest. For $\Omega$ bounded, $m=1$ and $p>1$ it was shown by Levine and Payne ([LP1]) in 1974 and by Walter $([\mathrm{Wa}])$ in 1975 that there are solutions which blow up in finite time. This means that

$$
\limsup _{t \rightarrow T} \max _{\bar{\Omega}} u(x, t)=\infty \quad \text { for some } T<\infty .
$$

The major questions that have been studied since then are:

1. For which values of $m, p$ does blow-up occur?

2. For which initial functions does blow-up occur?

3. Where are the blow-up points located?

4. With which rate (in $t$ ) does the solution approach the blow-up time?

1991 Mathematics Subject Classification: 35K60, 35K65, 35B40.

The paper is in final form and no version of it will be published elsewhere. 
5. What is the profile (in $x$ ) at the blow-up time?

6. Can blow-up in infinite time occur?

7. If $\Omega=\mathbb{R}_{+}^{N}$, what is the critical Fujita exponent?

Here we give a survey of answers (or partial answers) to the above questions and we also present some basic ideas under the simplest circumstances.

1. $m=1, \Omega=(0, \infty)$. We will assume throughout this section that $u_{0} \in C^{1}$ and $\lim _{x \rightarrow \infty} u_{0}(x)=0$.

1.1. If $p \leq 1$ then all solutions are global.

To see this it is sufficient to verify that

$$
v(x, t)=e^{\alpha^{2} t}\left(e^{-\alpha x}+c\right)
$$

is a supersolution if $c>0$ and $\alpha^{2}=(1+c)^{p}$ (cf. [GL. Remark 2.2]). Let us mention here that if $p<1$ then uniqueness fails to hold (cf. [DFL, Theorem 3.5]).

1.2. If $p>1$ then there are explicit selfsimilar solutions that blow up in finite time.

They are of the form

$$
u(x, t)=(T-t)^{-\lambda} f_{-}(\xi), \quad T>0, \lambda=\frac{1}{2(p-1)}, \xi=\frac{x}{\sqrt{T-t}},
$$

$f_{-}$is the unique bounded solution of

$$
\begin{aligned}
f_{-}^{\prime \prime}(\xi)-\frac{\xi}{2} f_{-}^{\prime}(\xi)-\lambda f_{-}(\xi) & =0, \quad \xi>0, \\
-f_{-}^{\prime}(0) & =f_{-}^{p}(0) .
\end{aligned}
$$

The function $f_{-}$is given explicitly in terms of degenerate hypergeometric functions (see [FQ, Lemma 3.1]) and it is not difficult to verify that $u$ has the following properties (cf. [DFL, Lemma 3.1]):

(i) $u_{t}>0$ in $(0, \infty) \times(0, T)$,

(ii) $u(x, T)=k x^{-2 \lambda}, k=\pi^{-1 / 2}\left(\lambda \frac{\Gamma^{p}(\lambda+1 / 2)}{\Gamma(\lambda+1)}\right)^{2 \lambda}$,

(iii) $x^{2 \lambda} u(x, t) \rightarrow k$ as $x \rightarrow \infty, 0 \leq t \leq T$.

Here $x=0$ is the only blow-up point and the blow-up rates in $t$ and $x$ are $(T-t)^{-\lambda}$ and $x^{-2 \lambda}$, respectively. We shall show that many solutions behave similarly.

1.3. If $u$ is a solution that blows up in the time $T$ and

$$
\beta=\inf _{x>0}-\frac{u_{0}^{\prime}(x)}{u_{0}^{p}(x)} \in(0,1]
$$

then

$$
\limsup _{t \rightarrow T} u(x, t) \leq[\beta(p-1)]^{-2 \lambda} x^{-2 \lambda} \quad \text { for } x>0 .
$$

This was shown in [B, Theorem 2].

The proof follows by a simple maximum principle argument. If we take

$$
J(x, t)=u_{x}+\beta u^{p}
$$


then it is not difficult to show that $J \leq 0$ in $\Omega \times(0, T)$. If we integrate the inequality

$$
u_{x}+\beta u^{p} \leq 0
$$

we obtain the assertion.

1.4. Assume $u$ blows up at the time $T$ and $u_{0}^{\prime} \leq 0$. Then there is a $\delta=\delta\left(u_{0}\right)>0$ such that

$$
\limsup _{t \rightarrow T} u(x, t) \geq p^{-\frac{p}{p-1}}(p-1) x^{-2 \lambda} \quad \text { for } x \in(0, \delta) .
$$

To show this one uses the intersection-comparison method as in [GKS]. Namely, for any $u_{0}$ there are $\alpha_{0}, \delta>0$ such that the stationary solution $U_{\alpha}(x)=-\alpha^{p} x+\alpha$ has in $(0, \delta)$ a unique intersection with $u_{0}$ for all $\alpha \geq \alpha_{0}$ and $U_{\alpha}(0)>u_{0}(0)$. Since $u_{x} \leq 0$ and $u$ blows up, we obtain that for any $\alpha \geq \alpha_{0}$ there is a $t_{\alpha} \in(0, T)$ such that $U_{\alpha}(0)<u\left(0, t_{\alpha}\right)$. The number of intersections is nonincreasing therefore it is actually equal to zero at $t=t_{\alpha}$. Hence $\lim \sup _{t \rightarrow T} u(x, t) \geq \sup _{\alpha>\alpha_{0}} U_{\alpha}(x)$ for $x \in(0, \delta)$ and it is easy to verify that $\sup _{\alpha \geq \alpha_{0}} U_{\alpha}(x)=p^{-p /(p-1)}(p-1) x^{-2 \lambda}$.

In 1.3 and 1.4 we described the profile in $x$ and next we turn to the same question but in $t$.

1.5. Assume $u_{0} \in C^{3},(-1)^{i} u_{0}^{(i)} \geq 0, i=1,2,3$ and $-u_{0}^{\prime \prime \prime}(0)=p u_{0}^{p-1}(0) u_{0}^{\prime \prime}(0)$. Then $u$ blows up at a finite time $T$ and

$$
u(0, t) \leq(p-1)^{-\lambda}(T-t)^{-\lambda} \quad \text { for } t \in(0, T) .
$$

We proceed as in [FQ, Lemma 2.1] (cf. also [DFL, Theorem 3.4]). By the maximum principle $u, u_{t} \geq 0$ and $u_{x}, u_{x t} \leq 0$. Using this and integration by parts we obtain

$$
\begin{aligned}
\frac{1}{2} u^{2 p}(0, t) & =\frac{1}{2} u_{x}^{2}(0, t)=-\int_{0}^{\infty} u_{x x}(x, t) u(x, t) d x \\
& =-\int_{0}^{\infty} u_{t}(x, t) u_{x}(x, t) d x \\
& =-\lim _{x \rightarrow \infty} u_{t}(x, t) u(x, t)+u_{t}(0, t) u(0, t)+\int_{0}^{\infty} u_{x t}(x, t) u(x, t) d x \\
& \leq u_{t}(0, t) u(0, t) .
\end{aligned}
$$

From the inequality

$$
u_{t}(0, t) \geq \frac{1}{2} u^{2 p-1}(0, t)
$$

we conclude that $u$ blows up at a time $T$ and integrating over $(t, T)$ we obtain the result.

1.6. Assume that $u_{0}^{\prime} \leq-u_{0}^{p}$ and $u$ blows up at a finite time $T$. Then

$$
u(0, t) \geq\left(\lambda p^{-1}\right)^{\lambda}(T-t)^{-\lambda} \quad \text { for } t \in(0, T) .
$$

We proceed similarly as in 1.3. By the maximum principle, $J(x, t)=u_{x}+u^{p} \leq 0$ in $\Omega \times(0, T)$ and $J(0, t)=0$. Therefore $J_{x}(0, t)=u_{t}(0, t)-p u^{2 p-1}(0, t) \leq 0$. Integration of the last inequality over $(t, T)$ yields the result. Notice that 1.5 and 1.6 give upper and 
lower bounds for $T$ in terms of $p$ and $u_{0}(0)$. As an example of a function $u_{0}$ satisfying all assumption in 1.5 and 1.6 we can take

$$
u_{0}(x)=\left[4 \lambda a(x+a)^{-2}\right]^{2 \lambda}, \quad a>0 .
$$

For the existence time $T$ of the solution starting from this initial function we obtain

$$
\frac{(p-1) a^{2}}{8 p} \leq T \leq \frac{(p-1) a^{2}}{4} .
$$

1.7. If $p>2$ then there are global selfsimilar solutions. They are of the form

$f_{+}$satisfies

$$
u(x, t)=\left(t_{0}+t\right)^{-\lambda} f_{+}(\zeta), \quad \zeta=\frac{x}{\sqrt{t_{0}+t}}, t_{0}>0
$$

$$
\begin{aligned}
f_{+}^{\prime \prime}(\zeta)+\frac{\zeta}{2} f_{+}^{\prime}(\zeta)+\lambda f_{+}(\zeta) & =0, \quad \zeta>0 \\
-f_{+}^{\prime}(0) & =f_{+}^{p}(0),
\end{aligned}
$$

and it can be expressed explicitly in terms of degenerate hypergeometric functions (cf. [DFL]).

1.8. If $p \in(1,2]$ then all solutions blow up in finite time. If $p>2$ then there are both global and nonglobal solutions. ( $p=2$ is the critical Fujita exponent.)

The first statement is shown by Kaplan type arguments in [GL]. The second one follows from 1.7 .

1.9. Assume $p>2$. Then the solution blows up in finite time provided

$$
\liminf _{x \rightarrow \infty} x^{2 \lambda} u_{0}(x) \geq k,
$$

$k$ is from $1.2(\mathrm{ii})$. On the other hand, there are global solutions such that $\lim _{x \rightarrow \infty} x^{2 \lambda} u(x, t)$ exists and is positive for all $t>0$.

The first assertion follows by comparison with selfsimilar solutions from 1.2. The property from the second statement is satisfied for a one parameter family of selfsimilar solutions from 1.7 (cf. [DFL]).

1.10. If $\Omega=\mathbb{R}_{+}^{N}$ then the Fujita type result from 1.8 holds with the critical exponent $p=2$ replaced by $p=1+1 / N($ cf. $[\mathrm{DFL}])$.

2. $m<1, \Omega=(0, \infty)$. Assume $\sup \left|\left(u_{0}^{m^{-1}-1}\right)^{\prime}\right|<\infty, u_{0}$ has compact support and $-u_{0}^{\prime}(0)=u_{0}^{p}(0)$.

2.1. If $p \leq(m+1) / 2$ then all solutions are global. If $p>(m+1) / 2$ then there are solutions that blow up in finite time.

2.2. If $p \in((m+1) / 2, m+1]$ then all solutions blow up in finite time. If $p>m+1$ then global solutions exist.

All statements in 2.1 and 2.2 were proved in [GL]. The most difficult and very interesting result here is blow-up of all solutions when $p=m+1$. All other results in 2.1 and 2.2 are proved by comparison with sub- and supersolutions of selfsimilar type. 


\section{3. $m=1, \Omega$ is a bounded domain in $\mathbb{R}^{N}$}

3.1. If $p \leq 1$ then all solutions are global. If $p>1$ then there are solutions that blow up in finite time.

Blow-up of solutions emanating from "large" initial data was established in [LP1] using energy methods. In [Wa] both the global existence and the blow-up result were shown by comparison arguments.

\subsection{If $p>1$ then all (positive) solutions blow up in finite time.}

We indicate here how this fact follows from the result in $[\mathrm{Fa}]$ (discussed below) which says that global solutions are bounded provided $p<N /(N-2)$ if $N>2$. It is easy to see that there are no positive steady states and that zero is unstable. If a solution were global then it would be bounded and its $\omega$-limit set would have to contain nonnegative steady states - a contradiction. If $p \geq N /(N-2)$ then a comparison argument finishes the proof. In [LMW1] this result was established for balls in $\mathbb{R}^{N}$ and simply connected domains in $\mathbb{R}^{2}$. See also $[\mathrm{HY}]$ for a short proof.

3.3. If $a \in \bar{\Omega}$ is a blow-up point then $a \in \partial \Omega$. (We call $a$ a blow-up point if there are $\left\{x_{n}\right\} \subset \Omega$ and $t_{n} \rightarrow T<\infty$ such that $x_{n} \rightarrow a$ and $\lim _{t \rightarrow T} u\left(x_{n}, t_{n}\right)=\infty$.)

This result was first proved for radially symmetric solutions in [LMW1] using a maximum principle argument similar as in 1.3. The general case was settled later in [HY] under the assumption that $u \leq C(T-t)^{-q}$ for some $C, q>0$. (This is satisfied for example if $\Delta u_{0} \geq 0$.)

3.4. There is an example of single point blow-up on the boundary.

This example can be found in [H2].

3.5. Assume that $\partial \Omega \in C^{2+\alpha}$ and $p<N /(N-2)$ if $N>2$. Suppose $u_{0} \in C^{2}(\bar{\Omega})$ and $\Delta u_{0} \geq 0$ in $\Omega$. Then

$$
\max _{\bar{\Omega}} u(x, t) \leq C(T-t)^{-\lambda}
$$

$\lambda=1 / 2(p-1)$ as in Section 1 .

This result was first established in the radially symmetric case (no restriction on $p$ is needed there) in $[\mathrm{FQ}]$ under additional assumptions on $u_{0}$ (cf. 1.5). In $[\mathrm{HY}]$ the general case was proved under a stronger restriction on $p$, namely, $p<(N-1) /(N-2)$ if $N>2$. This restriction was needed because of lack of a sharp nonexistence result for

$$
\begin{array}{ll}
\Delta u=0 & \text { in } \mathbb{R}_{+}^{N}, \\
\frac{\partial u}{\partial x_{1}}=u^{p} & \text { for } x_{1}=0 .
\end{array}
$$

The sharp nonexistence result was established later in [H1].

3.6. Suppose $\partial \Omega \in C^{1+\alpha}$. Then

$$
\max _{\bar{\Omega}} u(x, t) \geq c(T-t)^{-\lambda} \text {. }
$$

Using an integral representation of $u$, this was shown in $[\mathrm{HY}]$. 
3.7. Assume $\Omega=(-1,1), u_{0}(x)=u_{0}(-x)$ and $u_{0}^{(i)}(x) \geq 0, i=1,2,3,4, x \in[0,1]$. Let $T$ be the blow-up time. Then for any $y \geq 0$ we have

$$
(T-t)^{\lambda} u(1-y \sqrt{T-t}, t) \rightarrow f_{-}(y) \quad \text { as } t \rightarrow T
$$

uniformly on compact intervals; $f_{-}$is from 1.2 .

For the proof (also in the radial case on balls in higher dimension) we refer to [FQ]. For a generalization see $[\mathrm{HY}]$.

3.8. Suppose that $\partial \Omega \in C^{2+\alpha}$ and

$$
\max _{\bar{\Omega}} u(x, t) \leq C(T-t)^{-\lambda}
$$

for some $C>0$. If for some $K>0$

$$
\liminf _{t \rightarrow T}(T-t)^{\lambda} \inf _{|y| \leq K} u(a+y \sqrt{T-t}, t)=0,
$$

then a is not a blow-up point.

This nondegeneracy of the blow-up limit was established in [H2].

3.9. Let $u$ be a global solution of

$$
\begin{array}{lr}
u_{t}=\Delta u, & x \in \Omega, t>0, \\
\frac{\partial u}{\partial \nu}=f(u), & x \in \partial \Omega, t>0, \\
u(x, 0)=u_{0}(x), & x \in \bar{\Omega},
\end{array}
$$

with $\partial \Omega \in C^{2}$ and $f \in C^{\alpha}$ for some $\alpha \in(0,1)$. Suppose

$$
u f(u) \geq(2+\varepsilon) \int_{0}^{u} f(v) d v-C
$$

for some positive constants $\varepsilon, C$. Assume

(i) $|f(u)| \leq g(u)$ for some increasing $C^{1}$ function $g$ if $N=1$,

(ii) $|f(u)| \leq g(\vartheta) e^{\vartheta u^{2}}$ for some positive function $g$ and all $\vartheta>0, u \in \mathbb{R}$ if $N=2$,

(iii) $|f(u)| \leq \vartheta|u|^{N /(N-2)}+g(\vartheta)$ for some positive function $g$ and all $\vartheta>0, u \in \mathbb{R}$ if $N>2$.

Then $u$ is uniformly bounded in $C^{1, \alpha}$.

This was proved in $[\mathrm{L}]$. It is a significant improvement of the result from [Fa]. It says that under the above assumptions there are just two possible types of behavior of solutions:

(a) blow-up in finite time,

(b) global existence and uniform boundedness.

Blow-up in infinite time cannot occur. 


\section{4. $m>0, \Omega$ is a bounded domain in $\mathbb{R}^{N}$}

4.1. Assume $(N-2) m<N+2$. If $\lim _{\sup } \sup _{t \rightarrow T} \max _{\bar{\Omega}} u(x, t)=\infty$ then also

$$
\limsup _{t \rightarrow T} \oint_{\partial \Omega}|u(X, t)|^{r} d S=\infty \quad \forall r>(N-1)(p-1) .
$$

It was proved in [Fo1] (for a more general reaction term and with no sign restriction on $\left.u_{0}\right)$ that for any $r>(N-1)(p-1)$ there exist positive constants $M, \xi$, independent of $T$ such that

$$
|u(x, t)| \leq M\left(1+\sup _{\bar{\Omega}}\left|u_{0}(x)\right|\right)\left(1+\sup _{0 \leq \tau \leq t} \oint_{\partial \Omega}|u(X, \tau)|^{r} d S\right)^{\xi}
$$

$\forall(x, t) \in \bar{\Omega} \times[0, T)$ and the assertion follows.

The proof of the above estimate is based on Moser's iteration technique and it makes use of the inequalities:

$$
\begin{aligned}
& \oint_{\partial \Omega}|u|^{p+\lambda} \leq\left(\oint_{\partial \Omega}|u|^{\frac{(\lambda+1)(N-1)}{N-2}}\right)^{P}\left(\oint_{\partial \Omega}|u|^{(N-1)(p-1)+\varepsilon}\right)^{Q}\left(\oint_{\partial \Omega}|u|^{\lambda+1}\right)^{R}, \\
& P=\frac{(p-1)(N-2)}{(N-1)(p-1)+\varepsilon}, Q=\frac{p-1}{(N-1)(p-1)+\varepsilon}, R=\frac{\varepsilon}{(N-1)(p-1)+\varepsilon}
\end{aligned}
$$

if $N>2$, and

$$
\begin{gathered}
\oint_{\partial \Omega}|u|^{p+\lambda} \leq\left(\oint_{\partial \Omega}|u|^{2(\lambda+1)(p-1+\varepsilon) / \varepsilon}\right)^{P}\left(\oint_{\partial \Omega}|u|^{p-1+\varepsilon}\right)^{Q}\left(\oint_{\partial \Omega}|u|^{\lambda+1}\right)^{R}, \\
P=\frac{\varepsilon(p-1)}{(p-1+\varepsilon)(2(p-1)+\varepsilon)}, Q=\frac{p-1}{p-1+\varepsilon}, R=\frac{\varepsilon}{2(p-1)+\varepsilon}
\end{gathered}
$$

if $N=2(0<\varepsilon<\infty)$.

4.2. Assume $\Omega=(-1,1), u_{0}(x)=u_{0}(-x)$ and

(i) $0<m<1$.

If $p \leq m$ then each solution exists globally whereas in the case $m<p$ all solutions blow up in finite time. In the case $m<p \leq 1$ solutions become unbounded on the whole space interval $[-1,1]$, but for $p>1$ the only blow up points are $x= \pm 1$.

(ii) $m \geq 1$.

If $2 p \leq m+1$ then all solutions are global and for $2 p>m+1$ all solutions blow up in finite time.

All statements in 4.2 except for the case $2 p=m+1>2$ were proved in [Fo2]. The borderline case $2 p=m+1>2$ was settled later in [Wo] (see 4.3 below). The results are proved by comparison with solutions emanating from special chosen initial data (cf. [Fo2]). In some cases also the rate in $t$ and profile in $x$ at the blow-up time are shown.

If $0<m<p \leq 1$ and $u$ is a solution that blows up in the time $T$ such that $u_{x}, u_{x x}$ 
are nonnegative on $[0,1], u_{0}(1)>1$ then

$$
\xi^{p} \leq T(p-m) m^{-1}\left(\int_{0}^{1} u_{0}^{m}(x) d x\right)^{(p-m) / m} \leq 1,
$$

$\xi=1-u_{0}^{p-1}(1)$ and

$$
\xi^{(2 p-m) /(p-m)} \leq c(T-t)^{1 /(p-m)} u(x, t) \leq \xi^{-1}
$$

$\forall(x, t) \in[0,1] \times[0, T), c=((p-m) / m)^{1 /(p-1)}$.

If $0<m<1<p$ and $u$ is a solution such that $u_{x}$ is nonnegative on $[0,1]$ then

$$
u(x, t) \leq \frac{C}{(1-|x|)^{1 /(p-1)}}
$$

for some positive constant $C$.

If $2 p>m+1>2$ and $u$ is a solution that blows up at time $T$ such that $u_{x}, u_{x x}, u_{x x x}$ are nonnegative on $[0,1] \times[0, T)$ then

$$
\frac{C_{\varepsilon}}{(T-t)^{1 /(2 p-m-1+\varepsilon)}} \leq u(1, t) \leq \frac{C}{(T-t)^{1 /(2 p-m-1)}}
$$

for some positive constants $C, C_{\varepsilon}$ and $0<\varepsilon \ll 1$.

The results of [Fo2] were generalized by [Wo] in two ways. In [Wo] general nonlinearities are allowed and the domain is an $N$-dimensional ball or any simply connected smooth domain in $\mathbb{R}^{2}$.

4.3. The problem

$$
\begin{array}{ll}
u_{t}=\Delta \Phi(u) & \text { in } B_{R} \times(0, T), B_{R}=\left\{x \in \mathbb{R}^{N}:|x|<R\right\}, \\
\frac{\partial \Phi(u)}{\partial \nu}=f(u) & \text { on } S_{R} \times[0, T), S_{R}=\left\{x \in \mathbb{R}^{N}:|x|=R\right\}, \\
u(x, 0)=u_{0}(x)>0 & \text { in } B_{R},
\end{array}
$$

where $\Phi, f$ are increasing functions that are positive for $u$ positive together with their derivatives and which go to infinity as $u$ goes to infinity, was studied in [Wo]. It was shown that

(A) if $\Phi^{\prime}(u) \geq C>0$ and

(i) $f(u) /(1+u)$ is bounded then all solutions are global,

(ii) $\int^{\infty} d s / f(s)<\infty$ then every solution blows up in finite time,

(B) if $0<\Phi^{\prime}(u) \leq C$ and

(i) $\Phi$ is concave or $f(u) / \Phi(u)$ is nondecreasing and $\sqrt{\Phi^{\prime}(u)} f(u) / \Phi(u)$ is bounded then every solution exists globally,

(ii) $\Phi$ is concave, $\liminf _{u \rightarrow \infty} f(u) \sqrt{\Phi^{\prime}(u)} / \Phi(u)>0$ and

$$
\int^{\infty} \frac{\sqrt{\Phi^{\prime}(s)} d s}{f(s)}<\infty
$$

then each solution blows up in finite time. 


\section{Related problems}

5.1. The problem

$$
\begin{array}{lr}
u_{t}=\nabla(a(u) \nabla u), & x \in \Omega, t>0, \\
\frac{\partial u}{\partial \nu}=1, & x \in \partial \Omega, t>0, \\
u(x, 0)=u_{0}(x)>0, & x \in \bar{\Omega},
\end{array}
$$

was studied in [Y], where $a \in C^{1}$ is such that $a, a^{\prime}>0$ and $\limsup _{u \rightarrow \infty} a^{\prime}(u) / a(u)<\infty$. It was shown in $[\mathrm{Y}]$ that all solutions are global if and only if $\int^{\infty} d s / a(s)=\infty$. Also, some results on the profile near blow-up were established.

If we take $a(u)=m^{-1} u^{\frac{1}{m}-1}, 0<m \leq 1 / 2$ and $v=u^{1 / m}$ then $v$ satisfies

$$
\begin{array}{ll}
\left(v^{m}\right)_{t}=\Delta v, & x \in \Omega, t>0, \\
\frac{\partial v}{\partial \nu}=\frac{1}{m} v^{1-m}, & x \in \partial \Omega, t>0,
\end{array}
$$

which is a special case of $(0.1),(0.2)$ (if we neglect the factor $1 / m$ in $(5.5)$ ).

5.2. In $[\mathrm{WW}]$, the boundary condition (5.2) was replaced by

$$
\frac{\partial u}{\partial \nu}=b(u)
$$

and a global existence - global nonexistence result was proved.

5.3. In [LP2], the Laplace operator in (0.1) was replaced by an elliptic operator of order $2 k$, and (0.2) was changed to correspond to the elliptic operator. For that problem with $m=1$, a "large" data blow-up result was established.

5.4. In [LS], the homogeneous Dirichlet condition was prescribed on a part of the boundary and "large" data blow-up was shown for $m=1$.

5.5. In $[\mathrm{CFQ}],[\mathrm{LMW}]$ and $[\mathrm{Q}]$ the following problem with a damping term in the equation was considered:

$$
\begin{array}{ll}
u_{t}=\Delta u-a u^{p}, & x \in \Omega \subset \mathbb{R}^{N}, t>0, \\
\frac{\partial u}{\partial \nu}=u^{q}, & x \in \partial \Omega, t>0, \\
u(x, 0)=u_{0}(x) \geq 0, & x \in \bar{\Omega},
\end{array}
$$

with $\Omega$ bounded, $p, q>1$ and $a>0$. For $N=1$ it was shown in [CFQ] that

(i) if $p<2 q-1$ (or $p=2 q-1$ and $a<q$ ) then there are solutions which blow up in finite time,

(ii) if $p>2 q-1$ (or $p=2 q-1$ and $a>q$ ) then all solutions are global and bounded,

(iii) if $p=2 q-1$ and $a=q$ then all nontrivial solutions exist globally but they are not bounded, they tend (as $t \rightarrow \infty$ ) pointwise to a singular steady state.

The statements (i) and (ii) were proved in [CFQ] also for balls in higher dimension. But for a general domain $\Omega$ only some partial results can be found in [CFQ]. It was shown later in $[\mathrm{Q}]$ for a general domain $\Omega$ that 
(a) if $p<2 q-1$ (or $p=2 q-1$ and $a$ is small) then there are solutions which blow up in finite or infinite time,

(b) if $p>2 q-1$ then all solutions are global and bounded.

5.6. In $[\mathrm{DFL}]$ the following system was studied:

$$
\begin{array}{llr}
u_{t}=\Delta u, & v_{t}=\Delta v, & x \in \mathbb{R}_{+}^{N}, t>0, \\
-\frac{\partial u}{\partial x_{1}}=v^{p}, & -\frac{\partial v}{\partial x_{1}}=u^{p}, & x_{1}=0, t>0, \\
u(x, 0)=u_{0}(x) \geq 0, & v(x, 0)=v_{0}(x) \geq 0, & x \in \mathbb{R}_{+}^{N},
\end{array}
$$

with $p, q>0$. It was shown there (among other things) that blow-up may occur if and only if $p q>1$ and all nontrivial solutions blow up if and only if

$$
\max \left(\frac{p+1}{p q-1}, \frac{q+1}{p q-1}\right) \geq N
$$

When we referred to [DFL] in Section 1, we did that with the hope that interested readers will easily see how to modify the results (or proofs) in the easier scalar case.

5.7. In $[\mathrm{FL}]$ the authors studied the profile of solutions that quench on the boundary. They studied the problem

$$
\begin{array}{lr}
u_{t}=u_{x x}, & x \in(0,1), t>0, \\
u_{x}(0, t)=0, & t>0, \\
u_{x}(1, t)=-u^{-\beta}(1, t), & x \in[0,1],
\end{array}
$$

with $\beta>0$. Every solution of this problem reaches zero (quenches) in finite time.

5.8. The heat equation with a condition similar to (0.2) prescribed on a hypersurface $\Gamma$ in a bounded domain $\Omega$ was studied in [CY]. Sufficient condition for global existence and finite time blow-up were established there and also some results on the blow-up rate and blow-up set were proved.

5.9. Assume $0<m, r<\infty$. The problem

$$
\begin{array}{lr}
\left(|u|^{m-1} u\right)_{t}=\sum_{i=1}^{N}\left(\left|u_{x_{i}}\right|^{r-1} u_{x_{i}}\right)_{x_{i}} & x \in \Omega, t>0, \\
\nabla_{r} u \cdot \nu=f(u) & x \in \partial \Omega, t>0, \\
u(x, 0)=u_{0}(x), & u_{0} \in L^{\infty}(\Omega) \cup W_{r+1}^{1}(\Omega),
\end{array}
$$

where $\nabla_{r} u=\left(\left|u_{x_{1}}\right|^{r-1} u_{x_{1}}, \ldots,\left|u_{x_{N}}\right|^{r-1} u_{x_{N}}\right)$ and

$$
f(u) \operatorname{sign} u \leq L\left(|u|^{p}+1\right), \quad L \geq 0,0 \leq p<\infty,
$$

was studied in [Fo3]. It was shown that if

$$
q>\max \left\{1, \frac{N-1}{r}\right\} \max \{p-r, 0\}
$$

then there exists a positive function $\mathcal{F} \in C^{2}\left(\mathbb{R}_{+}^{2}\right)$ depending solely on the data and $q$ 
such that

$$
\|u(t)\|_{L^{\infty}(\Omega)} \leq \mathcal{F}\left(\left\|u_{0}\right\|_{L^{\infty}(\Omega)}, \underset{0 \leq \tau \leq t}{\operatorname{ess} \sup _{\partial \Omega}} \oint_{\partial \Omega}|u(X, \tau)|^{q} d S\right)
$$

for a.e. $t \in[0, T](\mathcal{F}(x, y) \rightarrow \infty$ if $y \rightarrow \infty)$.

The global existence result was proved under the following assumptions:

$$
p \leq \min \{m, r\} \quad \text { or } \quad r<p<\frac{r(m+1)}{r+1}
$$

and $p<p^{*}$, where $p^{*}=r(m+2)$ if $N=1$ and $p^{*}=r(N+\max \{p, m\}+1) / N$ if $N \geq 2$.

\section{References}

[B] F. V. Bunkin, V. A. Galaktionov, N. A. Kirichenko, S. P. Kurdyumov and A. A. Samarskiǔ, Localization in a nonlinear problem of ignition by radiation, Dokl. Akad. Nauk SSSR 302 (1988), 68-71.

[CY] J. M. Chadam and H. M. Yin, A diffusion equation with localized chemical reactions, Proc. Edinburgh Math. Soc. 37 (1994), 101-118.

[CFQ] M. Chipot, M. Fila and P. Quittner, Stationary solutions, blowup and convergence to stationary solutions for semilinear parabolic equations with nonlinear boundary conditions, Acta Math. Univ. Comen. 60 (1991), 35-103.

[DFL] K. Deng, M. Fila and H. A. Levine, On critical exponents for a system of heat equations coupled in the boundary conditions, Acta Math. Univ. Comenian. 63 (1994), 169-192.

[Fa] M. Fila, Boundedness of global solutions for the heat equation with nonlinear boundary conditions, Comment. Math. Univ. Carolin. 30 (1989), 479-484.

[FL] M. Fila and H. A. Levine, Quenching on the boundary, Nonlinear Anal. 21 (1993), 795-802.

[FQ] M. Fila and P. Quittner, The blowup rate for the heat equation with a nonlinear boundary condition, Math. Methods Appl. Sci. 14 (1991), 197-205.

[Fo1] J. Filo, Uniform bounds for solutions of a degenerate diffusion equation with nonlinear boundary conditions, Comment. Math. Univ. Carolin. 30 (1989), 485-495.

[Fo2] - Diffusivity versus absorption through the boundary, J. Differential Equations 99 (1992), 281-305.

[Fo3] - Local existence and $L^{\infty}$-estimate of weak solutions to a nonlinear degenerate parabolic equation with nonlinear boundary data, Panamerican Math. J. 4 (1994), $1-31$.

[GKS] V. A. Galaktionov, S. P. Kurdyumov and A. A. Samarskiŭ, On the method of stationary states for quasilinear parabolic equations, Math. USSR-Sb. 67 (1990), 449-471.

[GL] V. A. Galaktionov and H. A. Levine, On critical Fujita exponents for heat equations with a nonlinear flux condition on the boundary, preprint.

[H1] B. Hu, Nonexistence of a positive solution of the Laplace equation with a nonlinear boundary condition, Differential Integral Equations 7 (1994), 301-313.

[H2] - Nondegeneracy and single-point-blowup for solution of the heat equation with a nonlinear boundary condition, University of Notre Dame preprint No. 203 (1994). 
[HY] B. Hu and H. M. Yin, The profile near blowup time for solutions of the heat equation with a nonlinear boundary condition, Trans. Amer. Math. Soc. 346 (1994), 117135.

[LP1] H. A. Levine and L. E. Payne, Nonexistence Theorems for the heat equation with nonlinear boundary conditions and for the porous medium equation backward in time, J. Differential Equations 16 (1974), 319-334.

[LP2] H. A. Levine and L. E. Payne, Some nonexistence theorems for initial-boundary value problems with nonlinear boundary constraints, Proc. Amer. Math. Soc. 46 (1974), 277-284.

[LS] H. A. Levine and R. A. Smith, A potential well theory for the heat equation with a nonlinear boundary condition, Math. Methods Appl. Sci. 9 (1987), 127-136.

[L] G. Lieberman, Study of global solutions of parabolic equations via a priori estimates. Part I: Equations with principal elliptic part equal to the Laplacian, ibid. 16 (1993), 457-474.

[LMW1] J. López Gómez, V. Márquez and N. Wolanski, Blow up results and localization of blow up points for the heat equation and localization of blow up points for the heat equation with a nonlinear boundary condition, J. Differential Equations 92 (1991), 384-401.

[LMW2] J. López Gómez, V. Márquez and N. Wolanski, Global behavior of positive solutions to a semilinear equation with a nonlinear flux condition, IMA Preprint Series No. 810 (1991).

[Q] P. Quittner, On global existence and stationary solutions for two classes of semilinear parabolic problems, Comment. Math. Univ. Carolin. 34 (1993), 105-124.

[Wa] W. Walter, On existence and nonexistence in the large of solutions of parabolic differential equations with a nonlinear boundary condition, SIAM J. Math. Anal. 6 (1975), 85-90.

[WW] M. Wang and Y. Wu, Global existence and blow-up problems for quasilinear parabolic equations with nonlinear boundary conditions, ibid. 24 (1993), 1515-1521.

[Wo] N. Wolanski, Global behavior of positive solutions to nonlinear diffusion problems with nonlinear absorption through the boundary, ibid. 24 (1993), 317-326.

[Y] H. M. Yin, Blowup versus global solvability for a class of nonlinear parabolic equations, Nonlinear Anal., to appear. 